\title{
Editorial
}

\section{Coupled Network Systems and Their Collective Behavior}

\author{
Jinde Cao, ${ }^{1,2}$ Jianquan Lu, ${ }^{1}$ Ahmed Elaiw, ${ }^{2}$ Xinsong Yang, ${ }^{3}$ and Qiankun Song ${ }^{4}$ \\ ${ }^{1}$ Department of Mathematics, Southeast University, Nanjing 210096, China \\ ${ }^{2}$ Department of Mathematics, Faculty of Science, King Abdulaziz University, Jeddah 21589, Saudi Arabia \\ ${ }^{3}$ Department of Mathematics, Chongqing Normal University, Chongqing 401331, China \\ ${ }^{4}$ Department of Mathematics, Chongqing Jiaotong University, Chongqing 400074, China
}

Correspondence should be addressed to Jinde Cao; jdcao@seu.edu.cn

Received 12 February 2014; Accepted 12 February 2014; Published 13 May 2014

Copyright (C) 2014 Jinde Cao et al. This is an open access article distributed under the Creative Commons Attribution License, which permits unrestricted use, distribution, and reproduction in any medium, provided the original work is properly cited.

Coupled network systems (CNSs) consist of many small, inexpensive agents, in which each agent is capable of collecting signals, processing information, and communicating with neighboring agents. Due to their wide applications in many fields including mobile sensor networks and Unmanned Aerial Vehicles, CNSs have attracted many researchers from different areas of sciences and technologies, ranging from mathematics and physics to computer science. Node collaboration is the key for the success of CNSs due to the fact that each node itself is limited by communication range, power, and processing ability. In the process of the nodes' signal exchanging, an interesting aspect of the dynamics in CNSs is that certain types of globally collective behavior emerge from local interactions among the nodes. Such behavior arises ubiquitously in biological systems, ecosystems, and physical systems.

This special issue focuses on the dynamics of coupled network systems with their collective behavior and engineering applications, and it aims to bring together the most recent developments and knowledge in some related fields. Potential topics include, but are not limited to, (a) synchronization of complex dynamical networks, (b) consensus of multiagent systems, (c) stochastic resonance and some other collective behaviors, (d) network analysis and control, (e) social network analysis, and (f) network applications in different areas.

The response to this special issue on Coupled Network Systems and Their Collective Behavior was beyond our expectation. We received 38 papers in this research fields. All manuscripts submitted to this special issue went through a rigorous peer-refereeing process. Based on the reviewers' reports, twenty-four original research articles are finally accepted. The contents embrace the synchronization of complex networks under different constraints, Gossip consensus algorithm, Cucker-Smale flocking analysis, dynamic average consensus, and application of ranking algorithms on crime busting model.

It is certainly impossible to provide in this short editorial a more comprehensive description for all articles in this special issue. However, the guest editors sincerely hope that our efforts by compiling these articles can enrich our readers and inspire researchers with regard to the seemingly common but actually important issue of coupled network systems.

\section{Acknowledgments}

The guest editors would like to thank the authors who submitted papers for consideration and the reviewers whose comments are important for us to make the decisions. All the participants have made it possible to have a very stimulating interchange of ideas. Many thanks are also due to the editorial board members of this journal owing to their great support and help for this special issue.
Jinde Cao

Jianquan Lu

Ahmed Elaiw

Xinsong Yang

Qiankun Song 


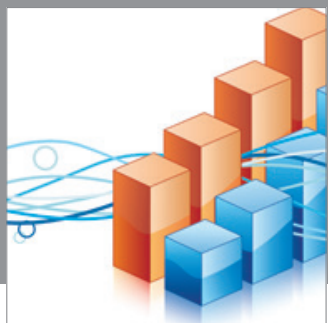

Advances in

Operations Research

mansans

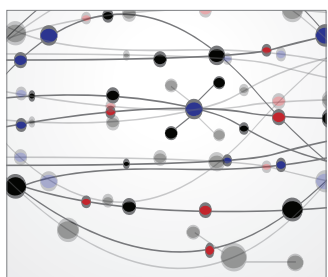

The Scientific World Journal
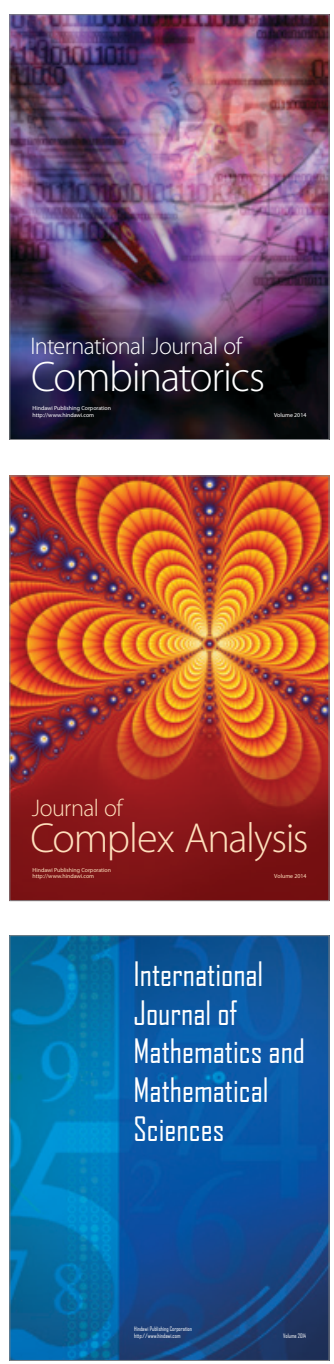
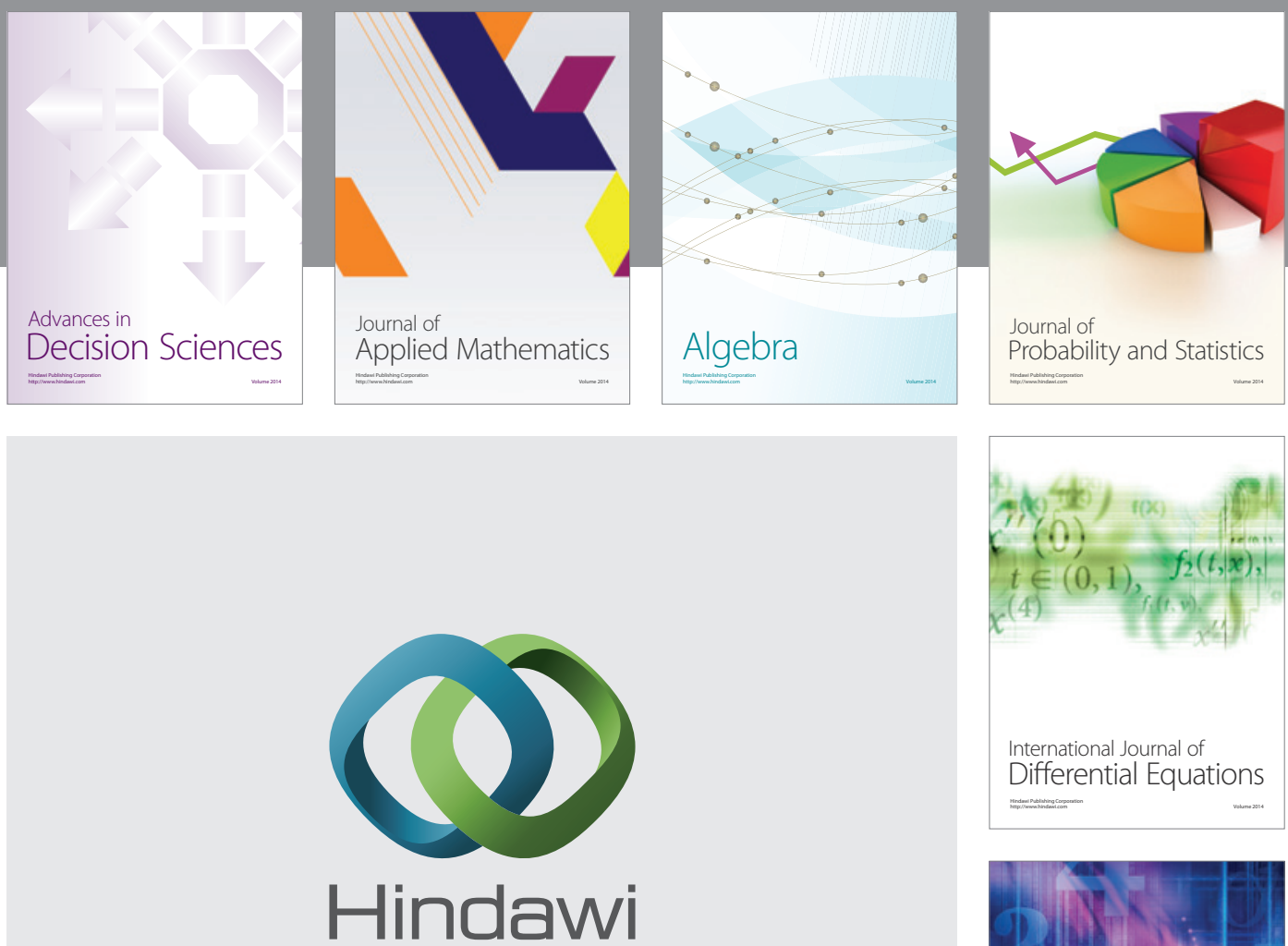

Submit your manuscripts at http://www.hindawi.com
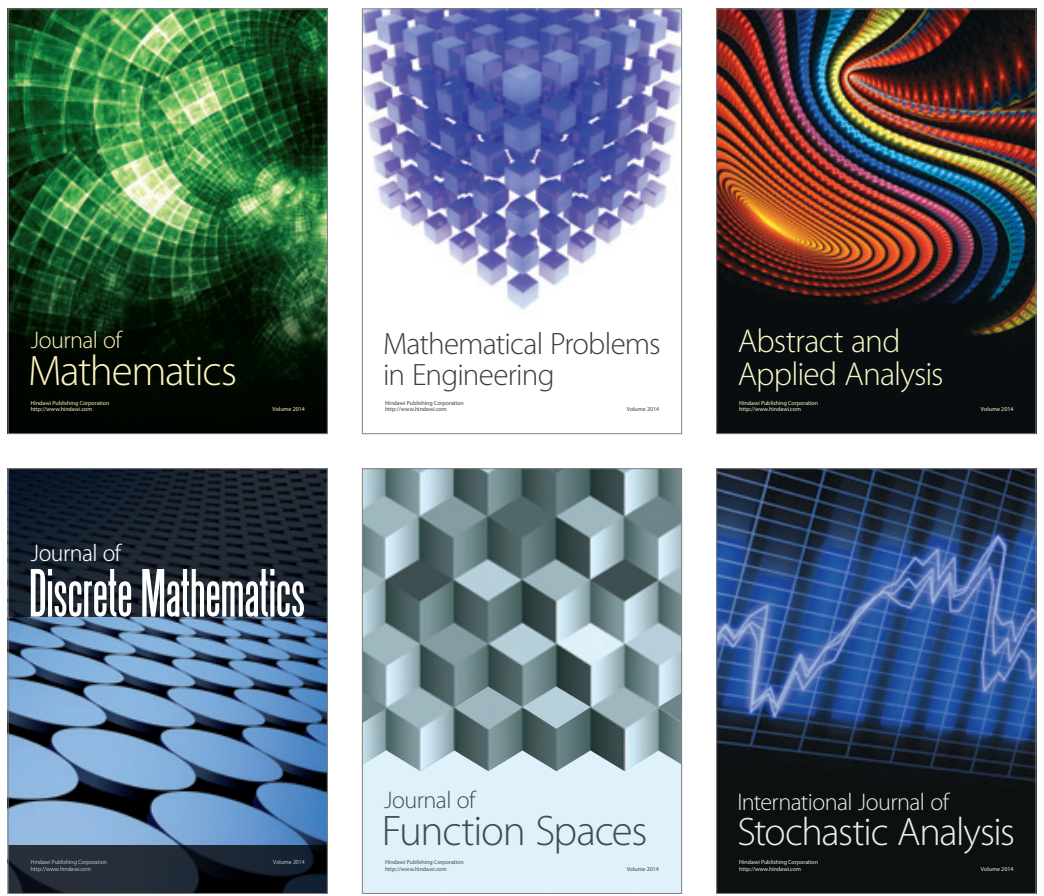

Journal of

Function Spaces

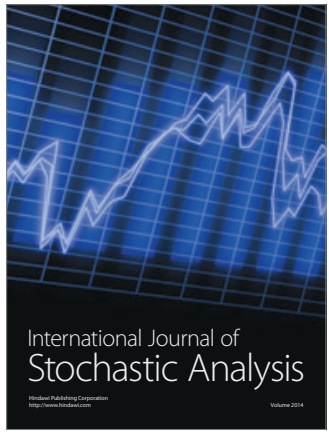

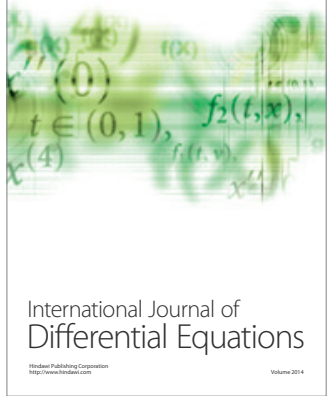
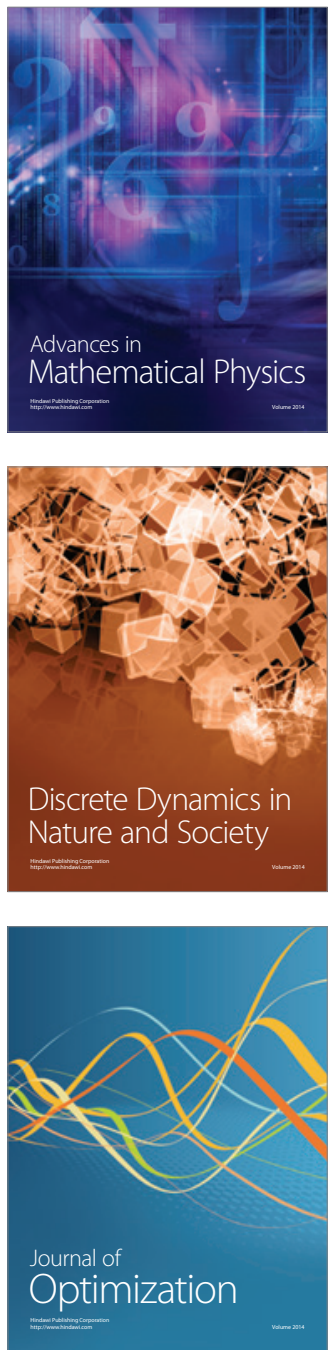